\title{
SOME RESULTS ON DEFORMATIONS OF SECTIONS OF VECTOR BUNDLES.
}

\author{
ABEL CASTORENA AND GIAN PIETRO PIROLA
}

\begin{abstract}
Let $E$ be a vector bundle on a smooth complex projective variety $X$. We study the family of sections $s_{t} \in H^{0}\left(E \otimes L_{t}\right)$ where $L_{t} \in \operatorname{Pic}^{0}(X)$ is a family of topologically trivial line bundle and $L_{0}=\mathcal{O}_{X}$, that is, we study deformations of $s=s_{0}$. By applying the approximation theorem of Artin [2] we give a transversality condition that generalizes the semi-regularity of an effective Cartier divisor. Moreover, we obtain another proof of the SeveriKodaira-Spencer theorem 4. We apply our results to give a lower bound to the continuous rank of a vector bundle as defined by Miguel Barja [3] and a proof of a piece of the generic vanishing theorems [6] and 7 for the canonical bundle. We extend also to higher dimension a result given in 8 on the base locus of the paracanonical base locus for surfaces.
\end{abstract}

\section{INTRODUCTION}

Let $X$ be a smooth complex projective variety of dimension $n$. Let $P=P i c^{0}(X)$ be the Picard variety of $X$. We recall that the tangent space to $P$ at any point is isomorphic to $H^{1}\left(X, \mathcal{O}_{X}\right)$. The irregularity of $X$ is $q(X):=\operatorname{dim} H^{1}\left(X, \mathcal{O}_{X}\right)=$ $\operatorname{dim} P$, and $X$ is said to be irregular if $q(X)>0$. Given a vector bundle $E$ on $X$ and $L \in P$, the action $(L, E) \rightarrow E \otimes L=E(L)$ defines a family of vector bundles parametrized by $P$. This paper is devoted to study the Brill-Noether loci:

$$
\mathcal{W}^{k}(E):=\left\{L \in P: h^{0}(E(L)) \geq k+1\right\}
$$

where $h^{i}(E)=\operatorname{dim} H^{i}(X, E)$. Set $\mathcal{W}^{0}(E)=\mathcal{W}(E)$. As in 9] we would like to give some conditions that implies that $\operatorname{dim} \mathcal{W}(E)>0$. This is a deformation problem for the sections of $E$. Given $s \in H^{0}(X, E)$ and $\zeta \in H^{1}\left(X, \mathcal{O}_{X}\right)$, denote by $\zeta \cdot s$ its cup product, then the first order condition to deform $s$ in the direction $\zeta$ is $\zeta \cdot s=$ $0 \in H^{1}(X, E)$, and in general there are higher obstructions. Our first result (See section 2 ) is that they vanishes under a transversality hypothesis that generalizes, in our context, the semi-regularity condition and the Koszul condition $[8]$ borrowed from the work of M. Green and R. Lazarsfeld [6]. For any $\zeta \in H^{1}\left(X, \mathcal{O}_{X}\right)$, define the complex

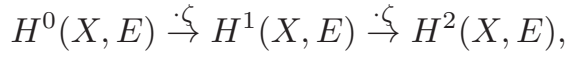

2000 Mathematics Subject Classification. Primary 14B12 and Secondary 14C20.

Key words and phrases. Continuous rank, paracanonical system, semi-regular divisor, vanishing theorems.

The first named author was partially supported by sabbatical Grants 232811(CONACyT, México) and PASPA-DGAPA (UNAM, México), and was partially supported as Visiting Professor by INdAM(Istituto Nazionale di Alta Matematica "F. Severi").

The second named author is partially supported by INdAM (GNSAGA); PRIN 2012 "Moduli, strutture geometriche e loro applicazioni" and FAR 2014 (PV) "Varietà algebriche, calcolo algebrico, grafi orientati e topologici". 
and set $H^{1}(X, E)_{\zeta}=\operatorname{ker}\left(\zeta: H^{1}(X, E) \rightarrow H^{2}(X, E)\right)$. From now on, we will denote by $\Delta \subset \mathbb{C}$ a complex disk with $0 \in \Delta$. Our first result is :

Proposition 1.1. (Transversality.) Let $s \in H^{0}(X, E)$ and $\zeta \in H^{1}\left(X, \mathcal{O}_{X}\right)$ be elements such that $\zeta \cdot s=0$ in $H^{1}(X, E)$. If $H^{1}(X, E)_{\zeta}=s \cdot H^{1}\left(X, \mathcal{O}_{X}\right)+\zeta$. $H^{0}(X, E)$ then there exists an analytic curve $\gamma: \Delta \rightarrow P$ such that $\gamma(0)=\mathcal{O}_{X}$ and $\gamma^{\prime}(0)=\zeta$, and there exists a family of sections $s(t) \in H^{0}(X, E(\gamma(t)))$ such that $s(0)=s$. In other words, the direction $\zeta$ is not obstructed for $s$. Moreover we may assume that the image of $\gamma$ contains an open set of an algebraic curve. In particular $\operatorname{dim} \mathcal{W}(E)>0$.

To prove our result we first solve the problem in the formal setting, then we apply the powerful approximation theorem of Artin (See 2], Theorem 1.2) that assure the existence of a convergent solution and finally the algebraic version, since the scheme $\mathcal{W}(E)$ is algebraic.

An important case of Proposition 1.1 is when $E$ is a line bundle and this is in connection with the semi-regularity. In fact, we remark in section 2.1 that an effective Cartier divisor $D$ which is semi-regular (See definition 2.4 below) satisfies $H^{1}(X, E)=H^{1}(X, E)_{\zeta}=s \cdot H^{1}\left(X, \mathcal{O}_{X}\right)$, where $E=\mathcal{O}_{X}(D), s \in H^{0}(X, E)$ is a section such that $D$ is the the zero-locus of $s$ and $\zeta \cdot s=0$ for some $\zeta$. The semiregularity gives also information on the infinitesimal structure of the Hilbert scheme $\mathcal{H}_{X, D}$ that parametrizes effective divisors on $X$ with fixed Chern class (See section 2 ). To be more precise, the Severi-Kodaira-Spencer theorem asserts that if $D$ is a semi-regular divisor, then $\mathcal{H}_{X, D}$ is smooth at $[D]$ (See e.g. [4 Theorem 1.2 part (i), 10, p. 157). In this context we give another proof of this theorem by applying the transversality condition of Proposition 1.1, in particular the semi-regularity is an open condition on the locus $\mathcal{W}(E) \subset \operatorname{Pic}(X)$.

To illustrate our second result (See section 3), following Miguel Barja (See [3]), we let

$$
r_{c}=r_{c}(E)=\min _{L \in P} h^{0}(E(L))
$$

be the continuous rank of $E$. Note that $r_{c}(E)>0 \Longleftrightarrow \mathcal{W}(E)=P$. We will see that $r_{c} \geq h^{0}(E)-h^{1}(E)$. This inequality should be well-known and it certainly will sound familiar to any expert on deformation theory. A stronger result will be given using the Koszul condition. We have :

Proposition 1.2. For any $\zeta \in H^{1}\left(X, \mathcal{O}_{X}\right)$, set $h^{1}(E)_{\zeta}=\operatorname{dim} H^{1}(X, E)_{\zeta}$. It holds $r_{c}(E) \geq h^{0}(E)-h^{1}(E)_{\zeta} \geq h^{0}(E)-h^{1}(E)$.

When $X$ is a smooth curve we have $h^{1}(X, E)=h^{1}(X, E)_{\zeta}$, and by RiemannRoch theorem the Proposition 1.2 gives $r_{c} \geq \chi(E)=c_{1}(E)-e(g-1)$. Thus for $e=1$, that is, when $E$ is a line bundle we obtain $\mathcal{W}(E)=P$ for $c_{1}(E) \geq g$, a version of the Jacobi inversion theorem.

In section 4 we consider the case when $E=\wedge^{k} \Omega_{X}^{1}=\Omega_{X}^{k}$ is the sheaf of holomorphic $k$-forms on $X$. Let $K_{X}=\Omega_{X}^{n}$ be the canonical sheaf on $X$. Using the $\partial \bar{\partial}$ - lemma (See [13], Proposition 6.17) we obtain a quick proof of a piece of the generic vanishing theorem of Green-Lazarsfeld. We give it by completeness, with not claim of originality. The approach is very similar to the one given by Clemens 
and Hacon in [5], but again we use the formal approximation theorem of Artin to show that the higher order obstructions vanish.

In section 5 we extend to higher dimensional varieties the result given in (8], Theorem 1.3) on the fixed part of the paracanonical base locus of surfaces. To explain this, let $P^{\kappa}$ be the isomorphism classes of line bundles algebraically equivalent to $K_{X}$. The morphism $L \rightarrow L \otimes K_{X}^{-1}$ gives an identification of $P^{\kappa}$ and $P$. Let $\operatorname{Div}^{\kappa}(X)$ be the projective variety that parametrizes effective divisors with class $\kappa, \operatorname{Div}^{\kappa}(X)$ is the paracanonical system of $X$. We consider the map $\rho: \operatorname{Div}^{\kappa}(X) \rightarrow P^{\kappa}, D \mapsto \mathcal{O}_{X}(D)$. The fiber $\rho^{-1}([L])$ is isomorphic to the linear system $|L|$. If $r_{c}\left(K_{X}\right)>0$, there is a unique irreducible component $\operatorname{Div}^{\kappa}(X)_{\text {main }}$ of $\operatorname{Div}^{\kappa}(X)$ that maps surjectively onto $P^{\kappa}$. The variety

$$
\operatorname{Div}^{\kappa}(X) \text { main }
$$

is called main component of $\operatorname{Div}^{\kappa}(X)$ of the paracanonical system.

We define the Albanese variety $A$ of $X$, to be the dual of $P: A=P i c^{0}(P)$. Let $a$ : $X \rightarrow A$ be the Albanese morphism (See [8]). We say that $X$ has maximal Albanese dimension if $\operatorname{dim} X=\operatorname{dim} a(X)$ and it is of Albanese general type if in addition $q=q(X)>\operatorname{dim} X$. We recall that generic vanishing theorems for the canonical bundle implies that $r_{C}\left(K_{X}\right)=\chi\left(K_{X}\right)$ for $X$ of maximal Albanese dimension (See 6]). If in addition $X$ is not fibred onto a variety $Y$ with $0<\operatorname{dim}(Y)<\operatorname{dim}(X)$ and with any smooth projective model $\widetilde{Y}$ of $Y$ of Albanese general type, then $\chi\left(K_{X}\right) \geq q-\operatorname{dim} X$. (See [11, Theorem A).

We define respectively the base locus $Z_{K}$ of the canonical system and $Z_{\kappa}$ the base locus of the main paracanonical component by:

$$
\begin{gathered}
Z_{K}=\left\{p \in X: p \in D \forall D \in\left|K_{X}\right|\right\} . \\
Z_{\kappa}=\left\{p \in X: p \in D \forall D \in \operatorname{Div}^{\kappa}(X)_{\text {main }}\right\} .
\end{gathered}
$$

Our result is the following:

Proposition 1.3. Let $X$ be a variety of Albanese general type with $\chi\left(K_{X}\right)>0$, then $Z_{\kappa} \subset Z_{K}$.

Acknowledgments. The authors give thanks to Rita Pardini and Miguel Angel Barja for fruitful discussions and suggestions. Special thanks are due to the referee for valuable comments which helped us to improve the manuscript.

The first named author give thanks to the Department of Mathematics of the University of Pavia for their warm hospitality and the use of their resources during a sabbatic year.

\section{Deformations of VECTOR BUndLES AND TRANSVERSALity}

Let $E \rightarrow X$ be a holomorphic vector bundle of rank $e$. Let $\mathcal{A}^{p, q}(E)$ be the sheaf of $\mathcal{C}^{\infty}$ forms of type $(p, q)$ with values on $E, \mathcal{C}^{\infty}(E)=\mathcal{A}^{0,0}(E)$. We have an operator

$$
\bar{\partial}_{E}: \mathcal{A}^{p, q}(E) \rightarrow \mathcal{A}^{p, q+1}(E)
$$

such that for any open subset $U \subset X$,

$$
U \rightarrow E(U)=\operatorname{ker} \bar{\partial}_{E \mid U}: \mathcal{C}^{\infty}(E)(U) \rightarrow \mathcal{A}^{0,1}(E)(U)
$$


gives the sheaf of holomorphic sections of $E$. The correspondence $E \leftrightarrow \bar{\partial}_{E}$ with the integrability condition is given by $\bar{\partial}_{E}^{2}=0$ (cf. e.g. [14]). A deformation of $E$ can be seen as a deformation of $\bar{\partial}_{E}$, that is, an operator $\bar{\partial}_{E}+T: \mathcal{A}^{p, q}(E) \rightarrow$ $\mathcal{A}^{p, q+1}(E)$ satisfying the integrability condition $\left(\bar{\partial}_{E}+T\right)^{2}=0$. Let $\gamma: \Delta \rightarrow P$ be an analytic curve in $P$ and assume that $\gamma(0)=\mathcal{O}_{X}$. The operators associated to the deformation $E(\gamma(t))=E \otimes \gamma(t), \gamma(t) \in P$, have a considerably simple linearization. To see this, consider the exponential map exp : $H^{1}\left(X, \mathcal{O}_{X}\right) \rightarrow P$, write $\gamma(t)=\exp (\alpha(t))$, where $\alpha: \Delta \rightarrow H^{1}\left(X, \mathcal{O}_{X}\right)$. We have $\alpha(t)=[v(t)]$, where $v(t) \in \mathcal{A}^{0,1}(X)$ is a $(0,1)$-form such that $\bar{\partial}(v(t))=0$. A canonical way to represent $\alpha(t)$ is to use the harmonic representative, that is, $v(t)=\bar{\omega}(t)$ where $\omega(t) \in H^{0}\left(X, \Omega_{X}^{1}\right)$. The corresponding family of operators on $E(\gamma(t))$ can be given by (See [7, p. 92)

$$
\begin{gathered}
\bar{\partial}_{E(\gamma(t))}=\bar{\partial}_{E}+\wedge v(t) . \\
\bar{\partial}_{E(\gamma(t))}(s)=\bar{\partial}_{E}(s)+s \wedge v(t) .
\end{gathered}
$$

The integrability conditions $\bar{\partial}_{E(\gamma(t))}^{2}=0$ is satisfied since $\bar{\partial} v(t)=0$ and $v(t) \wedge v(t)=$ 0 .

Given $\zeta_{1}=\left[v_{1}\right] \in H^{1}\left(X, \mathcal{O}_{X}\right)$ and $f_{0} \in H^{0}(X, E)$, the cup product $\left[v_{1} \cdot f_{0}\right] \in$ $H^{1}(X, E)$ gives the first order obstruction to deform $f_{0}$ in the $\zeta_{1}$-direction. In fact, if $\left[v_{1} \cdot f_{0}\right]=0$, we can find $f_{1} \in \mathcal{C}^{\infty}(E)$ such that $v_{1} \cdot f_{0}+\bar{\partial}_{E} f_{1}=0$, then

$$
\left(\bar{\partial}_{E}+t v_{1}\right)\left(f_{0}+t f_{1}\right)=t^{2}\left(v_{1} \cdot f_{1}\right)=0 \bmod t^{2} .
$$

Now we take sequences $f_{i} \in H^{0}(X, E), v_{i} \in \mathcal{A}^{0,1}\left(\mathcal{O}_{X}\right)$ satisfying $\bar{\partial} v_{i}=0$ and $\left[v_{i}\right] \in H^{1}\left(X, \mathcal{O}_{X}\right)$. We write the formal equation:

$$
\left(\bar{\partial}_{E}+t v_{1}+t^{2} v_{2}+\cdots+t^{n} v_{n}+\cdots\right) \cdot\left(f_{0}+t f_{1}+t^{2} f_{2}+\cdots+t^{n} f_{n}+\cdots\right)=0,
$$

in other words, such that the following is satisfied:

$$
\bar{\partial}_{E} f_{0}=0, \quad \bar{\partial}_{E} f_{1}+v_{1} f_{0}=0, \quad \bar{\partial}_{E} f_{2}+v_{1} f_{1}+v_{2} f_{0}=0,
$$

and in general:

$$
\bar{\partial}_{E} f_{n}+v_{1} f_{n-1}+\cdots+v_{k} f_{n-k}+\cdots+v_{n} f_{0}=0 .
$$

Definition 2.1. Fix $f_{0} \in H^{0}(X, E)$ and $\zeta_{1}=\left[v_{1}\right] \in H^{1}\left(X, \mathcal{O}_{X}\right)$. We say that the couple $\left(\zeta_{1}, f_{0}\right)$ allows a formal solution, if there exists a sequence $\left(v_{i}, f_{i}\right)$ satisfying the formal equation (4). We say moreover that the formal solution is linear if in (4) we can assume $v_{i}=0$ for $i>1$.

The following proposition is a consequence of the Artin's approximation theorem (See 2], Theorem 1.2).

Proposition 2.2. Let $f_{0} \in H^{0}(X, E)$ and $\zeta_{1} \in H^{1}\left(X, \mathcal{O}_{X}\right)$ be such that $\left(\zeta_{1}, f_{0}\right)$ allows a formal solution. Then there is an analytic curve $\gamma: \Delta \rightarrow P$ such that $\gamma(0)=\mathcal{O}_{X}$ and $\gamma^{\prime}(0)=\zeta_{1}$, and a family of sections $s(t) \in H^{0}(X, E(\gamma(t)))$ such that $s(0)=f_{0}$. In other words the direction $\zeta_{1}$ is not obstructed for $s$. In particular $\operatorname{dim} \mathcal{W}(E)>0$ and we may assume that the image of $\gamma$ contains an open set of an algebraic curve. 
Proof. We take a Zariski open set $W$ of $P$ that contains the origin $\mathcal{O}_{X}$, that is, there is an embedding $\pi: W \hookrightarrow \mathbb{C}^{N}$ such that the ideal of $\pi(\mathcal{W}(E)) \subset \mathbb{C}^{N}$ is generated by polynomial equations $\left\{F_{j}\right\}$. The formal curve

$$
\tilde{\gamma}(t)=\exp \left(\sum_{i=1}^{\infty} \zeta_{i} t^{i}\right)
$$

satisfies the equations $F_{i}(\tilde{\gamma}(t)) \equiv 0$. By $([2]$, Theorem 1.2 for $c=1)$ we can find an analytic curve $\gamma: \Delta \rightarrow P, \gamma(0)=\mathcal{O}_{X}$ and $\gamma^{\prime}(0)=\zeta_{1}$. The last sentence follows again since the $F_{j}$ are polynomials.

We give the following

Lemma 2.3. Suppose that for $\zeta_{i}=\left[v_{i}\right]$ and $i \leq n$, we have solved equation (5) in the $n$-th step, that is,

$$
\bar{\partial}_{E} f_{n}+v_{1} f_{n-1}+\cdots+v_{k} f_{n-k}+\cdots+v_{n} f_{0}=0 .
$$

Let $y=v_{1} f_{n}+v_{2} f_{n-1}+\cdots+v_{k} f_{n-k+1}+v_{n} f_{1}$ be, then

(1) $\bar{\partial}_{E}(y)=0$

(2) $v_{1} \wedge y \in \operatorname{Im} \bar{\partial}_{E}$

Proof. Since $\bar{\partial} v_{i}=0$, then $\bar{\partial}_{E}\left(v_{i} f\right)=v_{i} \wedge \bar{\partial}_{E}(f)$. Define $d=\bar{\partial}_{E}+t v_{1}+\cdots+$ $t^{n} v_{n}$. This is an operator $d: \mathcal{A}^{p, q}(E)[t] \rightarrow \mathcal{A}^{p, q+1}(E)[t]$ satisfying $d^{2}=0$, where $\mathcal{A}^{p, q}(E)[t]:=\mathcal{A}^{p, q}(E) \otimes \mathbb{C}[t]$ and $\mathbb{C}[t]$ is the ring of complex polynomials. Taking $f=f_{0}+t f_{1}+\cdots+t^{n} f_{n} \in \mathcal{A}^{0,0}(E)[t]=\mathcal{C}^{\infty}(E)[t]$, we get $d f=0 \bmod t^{n}$ by our assumption. Making some computations we have that

$$
d f=t^{n+1} y+t^{n+2} y_{1}+\cdots
$$

where $y=v_{1} f_{n}+v_{2} f_{n-1}+\cdots+v_{k} f_{n-k+1}+v_{n} f_{1}$. Now we have $d^{2}(f)=0$, thus

$$
0=d\left(y+t y_{1}+\cdots\right)=\left(\bar{\partial}_{E}+t v_{1}+\cdots+t^{n} v_{n}\right)\left(y+t y_{1}+\cdots\right)
$$

the first terms gives

$$
\bar{\partial}_{E}(y)=0, v_{1} \wedge y+\bar{\partial}_{E}\left(y_{1}\right)=0 .
$$

Now we prove the Proposition 1.1

Proof. Suppose that $\left(\zeta_{1}=\left[v_{1}\right], f_{0}\right)$ satisfies the transversal condition: $\zeta_{1} H^{0}(X, E)+$ $f_{0} H^{1}\left(X, \mathcal{O}_{X}\right)=H^{1}(X, E)_{\zeta_{1}}$. Moreover, assume that we have solved the equation (5]) in the $n$-th step, that is,

$$
\bar{\partial}_{E} f_{n}+\sum_{i=1}^{n} v_{i} f_{n-i}=0 .
$$

We would like to solve the $(n+1)$ th step. The proposition will follow by induction. Define $y=\sum_{i=1}^{n} v_{i} f_{n+1-i}$, we know that $\bar{\partial}_{E} y=0, \zeta_{1} \cdot[y]=\left[v_{1} \cdot y\right]=0 \in H^{2}(X, E)$ by the above lemma. Let $[y] \in H^{1}(X, E)_{\zeta_{1}}$ be its class. By the transversal hypothesis there are $\zeta_{n+1} \in H^{1}\left(X, \mathcal{O}_{X}\right)$ and $\tilde{s} \in H^{0}(X, E)$ such that

$$
[y]=\zeta_{1} \cdot \tilde{s}+\zeta_{n+1} f_{0},
$$


that is, choosing a representative $v_{n+1}$ for $\zeta_{n+1}, y=v_{n+1} f_{0}+v_{1} \tilde{s}+\bar{\partial}_{E} g$. Letting $f_{n}^{\prime}=f_{n}-\tilde{s}$ we solve again the $n$-th step equation

$$
\bar{\partial}\left(f_{n}-\tilde{s}\right)+\sum_{i=1}^{n} v_{i} f_{n-i}=\sum_{i=1}^{n} v_{i} f_{n-i}=0 .
$$

Then letting $f_{n+1}=-g$ we solve also the $(n+1)$-th step:

$$
\bar{\partial}\left(f_{n+1}\right)+v_{1}\left(f_{n}-\tilde{s}\right)+v_{2} f_{n-1}+\cdots+v_{n} f_{1}+v_{n+1} f_{0} .
$$

We see that $f_{0}, \ldots, f_{n-1}, f_{n}^{\prime}=f_{n}-\tilde{s}, f_{n+1}$ stabilizes in the first $n-1$ elements. Then we can find a formal solution and by (2.2) we prove our result.

2.1. Semi-regularity. Let $X$ be a smooth complex projective variety. The semiregularity of an effective Cartier divisor $D \subset X$ is an important example which satisfies the transversality condition of Proposition 1.1 for $E=L=\mathcal{O}_{X}(D)$, and is related also with the problem to determinate when a couple $(s, v)$ is non-obstructed in the sense of Proposition 2.2. To see this, let $s \in H^{0}(X, L)$ be a section such that $(s)_{0}=D$ is the zero-locus of $s$ and let $N_{D}=\left.L\right|_{D}$ the normal sheaf of $D$ in $X$. The normal bundle exact sequence $0 \rightarrow \mathcal{O}_{X} \stackrel{\cdot s}{\rightarrow} L \rightarrow N_{D} \rightarrow 0$ induces an exact sequence in cohomology

$\cdots \rightarrow H^{0}\left(D, N_{D}\right) \stackrel{\delta_{D}}{\longrightarrow} H^{1}\left(X, \mathcal{O}_{X}\right) \stackrel{\cdot s}{\rightarrow} H^{1}(X, L) \rightarrow H^{1}\left(D, N_{D}\right) \stackrel{\delta^{1}}{\rightarrow} H^{2}\left(X, \mathcal{O}_{X}\right) \rightarrow \cdots$

Definition 2.4. The divisor $D$ is said to be semi-regular if $H^{1}(X, L) \rightarrow H^{1}\left(D, N_{D}\right)$ is the zero-map.

Let $\mathcal{H}_{X, D}$ be the Hilbert scheme that parametrizes effective divisors $B$ on $X$ such that $c_{1}\left(\mathcal{O}_{X}(B)\right)=c_{1}\left(\mathcal{O}_{X}(D)\right)$. Is well known that first-order deformations $\tilde{D} \subset$ $X \times \operatorname{Spec}\left(\frac{k[t]}{\left(t^{2}\right)}\right)$ of $D$ in $X$ are in one-to-one correspondence with global sections $\tilde{s}$ in $H^{0}\left(D, N_{D}\right)$, where $H^{0}\left(D, N_{D}\right)$ is the Zariski tangent space $T_{[D]}\left(\mathcal{H}_{D, X}\right)$ to $\mathcal{H}_{X, D}$ at $[D]$ (See [12, Proposition 3.2.1). The obstruction to lifting $\tilde{D}$ to a deformation over $\operatorname{Spec}\left(\frac{k[t]}{\left(t^{n}\right)}\right)$ for some $n \geq 3$ determines a cohomological class $o b(\tilde{s})$ in $H^{1}\left(D, N_{D}\right)$ (See $[12$, p. 131). If $o b(\tilde{s}) \neq 0$, then $\tilde{s}$ is called obstructed, otherwise it is unobstructed. The set of obstructions define a subspace in $H^{1}\left(D, N_{D}\right)$, and this obstruction space is contained in the kernel of the semi-regular map $H^{1}\left(D, N_{D}\right) \stackrel{\delta^{1}}{\rightarrow} H^{2}\left(X, \mathcal{O}_{X}\right)$ (See e.g. 44). In particular when $\delta^{1}$ is injective the section $\tilde{s}$ is non-obstructed.

Note that for $t \in H^{0}\left(X, N_{D}\right)=T_{[D]}\left(\mathcal{H}_{X, D}\right)$ and $v=\delta_{D}(t) \in \operatorname{Im}\left(\delta_{D}\right) \subseteq H^{1}\left(X, \mathcal{O}_{X}\right)$ we have that $s \cdot v=0$. From the exact sequence (6), semiregularity for $D$ is equivalent to have $H^{1}(X, L)=s \cdot H^{1}\left(X, \mathcal{O}_{X}\right)$, and by properties of cap product we have $v \cdot H^{1}(X, L)=v \cdot\left(s \cdot H^{1}\left(X, \mathcal{O}_{X}\right)\right)=\{0\} \subset H^{2}(X, L)$, then $H^{1}(X, L)_{v}=$ $\operatorname{Ker}\left(H^{1}(X, L) \stackrel{v}{\rightarrow} H^{2}(X, L)\right)=s \cdot H^{1}\left(X . \mathcal{O}_{X}\right)=H^{1}(X, L)$, thus the transversal condition of Proposition 1.1 is satisfied for the couple $(s, v)$.

The following theorem was first claimed by Severi and proved by KodairaSpencer (See e.g. [4] Theorem 1.2 part (i), [10], p. 157). We give a proof of such theorem by applying the transversality criteria of Proposition 1.1 .

Theorem 2.5. (Severi-Kodaira-Spencer). Let $X$ be a complex smooth projective variety. Let $D \subset X$ be an effective Cartier divisor and set $L=\mathcal{O}_{X}(D)$. If $D$ is semiregular, then Hilbert scheme $\mathcal{H}_{X, D}$ is smooth at $[D]$ of the expected dimension $h^{0}(X, L)-h^{1}(X, L)-1+h^{1}\left(X, \mathcal{O}_{X}\right)$. 
Proof. As we have seen, the semiregularity is equivalent to the fact that the map $H^{1}\left(X, \mathcal{O}_{X}\right) \stackrel{\cdot s}{\rightarrow} H^{1}(X, L)$ is surjective, and in this case we have the transversality condition of Proposition 1.1 for couples $(s, w)$ for every $w \in \operatorname{Im}\left(\delta_{D}\right)=$ $\operatorname{Ker}\left(H^{1}\left(X, \mathcal{O}_{X}\right) \stackrel{\cdot s}{\rightarrow} H^{1}(X, L)\right)$. Since semiregularity is an open condition on the sections $s$, the dimension of the Hilbert scheme $\mathcal{H}_{X, D}$ is precisely $h^{0}(X, L)-1+$ $\operatorname{dim} \operatorname{Im}\left(\delta_{D}\right)$ and the Severi-Kodaira-Spencer theorem follows.

2.2. Examples. We give some examples where the Transversal condition of Proposition 1.1 is satisfied. In these examples $C$ will denote a non-hyperelliptic curve of genus $g \geq 3$.

(a).- Let $p \in C$ be a general point and let $u$ be a general extension in $\operatorname{Ext}^{1}\left(K_{C}, \mathcal{O}_{C}(p)\right) \simeq$ $H^{1}\left(C, T_{C}(p)\right)$, then the correponding coboundary map $\partial_{u}: H^{0}\left(C, K_{C}\right) \rightarrow H^{1}\left(C, \mathcal{O}_{C}(p)\right)=$ $\left(H^{0}\left(C, K_{C}(-p)\right)\right)^{\vee}$ is surjective, so there exists on $C$ a rank two vector bundle $E$ fitting in an exact sequence $0 \rightarrow \mathcal{O}_{C}(p) \rightarrow E \rightarrow K_{C} \rightarrow 0$ such that $h^{0}(C, E)=2$.

Claim. We can find two linearly independent sections $s, t$ in $H^{0}(C, E)$ such that $s(p)=0$ and $t(p) \neq 0$.

Proof of Claim. We will show that for the general extension in $\operatorname{Ext}^{1}\left(K_{C}(-p), \mathcal{O}_{C}\right)$ the corresponding coboundary map $H^{0}\left(C, K_{C}(-p)\right) \rightarrow H^{1}\left(C, \mathcal{O}_{C}\right)$ is injective.

Let $\eta \in \operatorname{Ext}^{1}\left(K_{C}(-p), \mathcal{O}_{C}\right) \simeq H^{1}\left(C, T_{C}(p)\right)$ and let $0 \neq \omega \in H^{0}\left(C, K_{C}(-p)\right)$ such that the cup product $\eta \cdot \omega=0$. Let $\Lambda_{\omega}:=\left\{\eta \in H^{1}\left(C, T_{C}(p)\right): \omega \cdot \eta=0\right\}$ and set $Z:=$ $Z(\omega)$ the divisor of $\omega$ which is of degree $2 g-3$ and let $\mathbb{P}:=\mathbb{P}^{g-2}=\mathbb{P}\left(H^{0}\left(C, K_{C}(p)\right)\right)$. Tensoring by $T_{C}(p)$ the exact sequence $\left.0 \rightarrow \mathcal{O}_{C} \stackrel{\omega}{\rightarrow} K_{C}(-p) \rightarrow K_{C}(-p)\right|_{Z} \rightarrow 0$ we have $\left.0 \rightarrow T_{C}(p) \stackrel{\omega}{\rightarrow} \mathcal{O}_{C}(p) \rightarrow \mathcal{O}_{C}(p)\right|_{Z} \rightarrow 0$. Taking cohomology one has that $\operatorname{dim}\left(\mathbb{P}\left(\Lambda_{\omega}\right)\right)=2 g-5$ and $\operatorname{dim} \operatorname{Span}\left(\bigcup_{\omega \in \mathbb{P}} \mathbb{P}\left(\Lambda_{\omega}\right)\right) \leq 2 g-5+g-2=3 g-7<$ $3 g-5=\operatorname{dim}\left(\mathbb{P}\left(H^{1}\left(C, T_{C}(p)\right)\right)\right)$. This proves the claim.

Now consider the following diagram

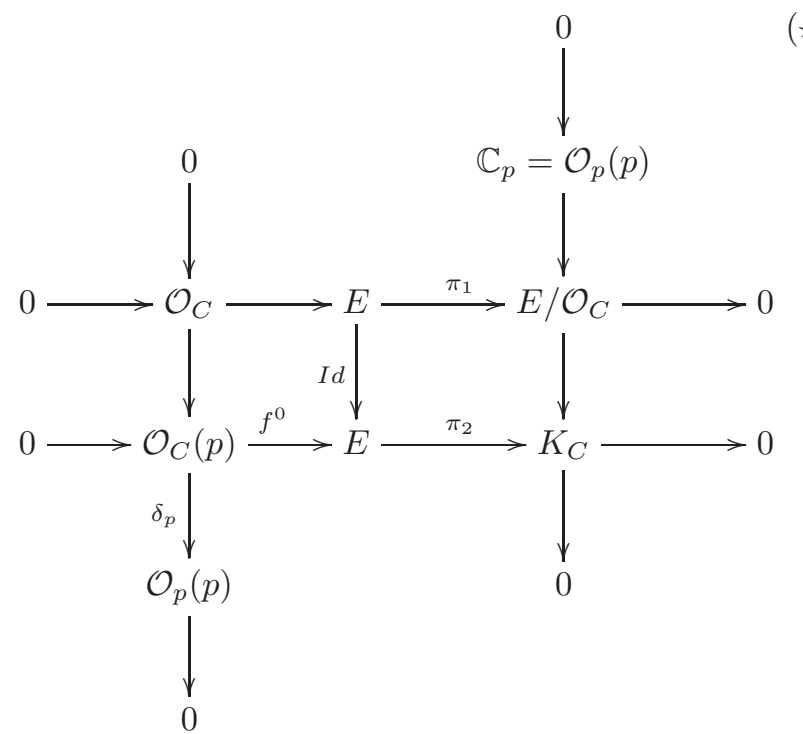


Let $v=v_{p}$ be a non-zero element in $\operatorname{Im}\left(\delta_{p}\right) \subset H^{1}\left(C, \mathcal{O}_{C}\right)$. From $(\star)$ we have an square diagram in cohomology :

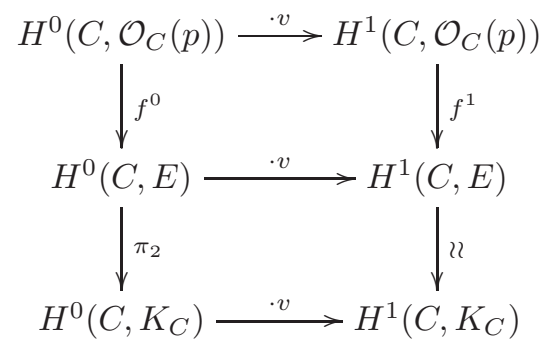

where $f^{0}, f^{1}$ are induced maps in cohomology. Let $u \in H^{0}\left(C, \mathcal{O}_{C}(p)\right)$ be a non-zero generator. Since $f^{0}$ is injective, the section $u \in H^{0}\left(C, \mathcal{O}_{C}(p)\right)$ can be identified with $f^{0}(u)=s \in H^{0}(C, E)$, then $v \cdot s=0=v \cdot f^{0}(s)=f^{1}(v \cdot u) \in H^{1}(C, E)$. For the other side, the section $t \in H^{0}(C, E)$ satisfies that $w:=\pi_{2}(t) \neq 0$ in $H^{0}\left(C, K_{C}\right)$ and $0 \neq v \cdot w \in H^{1}\left(C, K_{C}\right)$, then $v \cdot t \neq 0$ in $H^{1}(C, E)$, that is, we see that $v \cdot H^{0}(C, E) \neq 0$. Since $h^{1}(C, E)=1$, we have the transversal condition $v \cdot H^{0}(C, E)+s \cdot H^{1}\left(C, \mathcal{O}_{C}\right)=H^{1}(C, E)$.

(b).- Let $S=S^{2}(C)$ be the second symmetric product of $C$. For every $p \in S$ we have a natural divisor $X_{p}=\{p+q: q \in C\}$ in $S$. Let $N^{1}(S)_{\mathbb{Z}}$ be the Néron-Severi group of $S$ of numerically equivalent classes of divisors on $C^{(2)}$. Let $x$ be the class of $X_{p}$ in $N^{1}(S)_{\mathbb{Z}}$. This class is an ample class and is independent of of the point $p$. Let $\delta^{\prime}$ be the class in $N^{1}(S)_{\mathbb{Z}}$ of the diagonal divisor $\Delta=\{q+q: q \in C\} \subset S$. If $C$ has very general moduli, it is known that $N^{1}(S)_{\mathbb{Z}}$ is generated by the numerical equivalence classes $x$ and $\delta=\frac{\delta^{\prime}}{2}$ (See e.g. 11, p. 359). The intersection numbers between these numerical classes are $(x, x)=x^{2}=1,(x, \delta)=x \cdot \delta=1,(\delta, \delta)=(\delta)^{2}=1-g$.

For a non-hyperelliptic curve $C$ of genus three consider the divisor $D:=K_{S}-x=$ $3 x-\delta$ and the line bundle $L=\mathcal{O}_{S}(D)$. Let $s_{D}$ be the section of $L$ such that $D$ is the zero-locus of $s_{D}$. We have that $h^{0}(S, L)=1, \chi(L)=0$ and $h^{2}(S, L)=$ $h^{0}\left(S, \mathcal{O}_{S}(x)\right)=1$, then $h^{1}(S, L)=h^{0}(S, L)+1=2$. For the normal bundle $N_{D}$ we have $\operatorname{deg}\left(N_{D}\right)=D^{2}=1$, so $h^{0}\left(N_{D}\right)=1$. Since $h^{0}(S, L)=h^{0}\left(S, \mathcal{O}_{S}\right)$, we have $0 \rightarrow H^{0}\left(S, N_{D}\right) \stackrel{\delta}{\rightarrow} H^{1}\left(S, \mathcal{O}_{S}\right) \stackrel{\cdot s_{D}}{\rightarrow} H^{1}(S, L) \rightarrow H^{1}\left(S, N_{D}\right) \rightarrow \cdots$. Let $0 \neq w \in$ $H^{0}\left(N_{D}\right)$ be and let $v=\delta(w)$, we have that $v \cdot s_{D}=0$. Since $\cdot s_{D}$ is surjective, $D$ is semiregular and the transversal condition $v \cdot H^{0}(S, L)+s_{D} \cdot H^{1}\left(S, \mathcal{O}_{S}\right)=H^{1}(S, L)$ is clear.

\section{Continuous Rank}

Now we study the continuous rank of a vector bundle defined $E$ as in (1). Fixing $\zeta \in H^{1}\left(X, \mathcal{O}_{X}\right)$, we first define

$$
W_{\zeta}:=\left\{f \in H^{0}(X, E):(\zeta, f) \text { allows a formal linear solution }\right\}
$$

We remark that $W_{\zeta}$ is a subvector space of $H^{0}(X, E)$ because we consider only formal linear operators (see definition 2.1).

We will give an estimate of the dimension of $W_{\zeta}$. We recall that $H^{1}(X, E)_{\zeta}$ has been defined as the kernel of the map $\zeta: H^{1}(X, E) \rightarrow H^{2}(X, E)$ and $h^{1}(E)_{\zeta}=$ $\operatorname{dim} H^{1}(X, E)_{\zeta}$ its dimension. We consider the lines in $H^{1}\left(X, \mathcal{O}_{X}\right)$ given by $t \mapsto t \zeta$ and the corresponding curves $\gamma(t)=\exp (t \zeta)$ in $P$. If $v$ is a representative of $\zeta$ we also consider the operator $\bar{\partial}_{E}+t v$. 
Lemma 3.1. $\operatorname{dim} W_{\zeta} \geq h^{0}(E)-h^{1}(E)_{\zeta}$.

Proof. For the operator $\bar{\partial}_{E}+t v$ the equations (4) become

$$
\bar{\partial}_{E} f_{n}+v f_{n-1}=0 \text {. }
$$

Lemma 2.3 gives just $\bar{\partial}_{E}\left(v \cdot f_{i}\right)=v \wedge v \cdot f_{i}=0$.

First step. Consider the linear map

$$
\begin{gathered}
v^{(1)}: H^{0}(X, E) \rightarrow H^{1}(X, E)_{\zeta}, \\
f_{0} \mapsto\left[v f_{0}\right] .
\end{gathered}
$$

This is just the cup product map.

Let $K_{1}^{0}(v):=\operatorname{ker}\left(v^{(1)}\right), K_{1}^{1}(v):=\operatorname{Coker}\left(v^{(1)}\right)=H^{1}(X, E)_{\zeta} / \operatorname{Im}\left(v^{(1)}\right)$. Since $\operatorname{dim} \operatorname{ker}\left(v^{(1)}\right)=h^{0}(X, E)-\operatorname{dim} \operatorname{Im}\left(v^{(1)}\right)$, then

$$
\operatorname{dim} K_{1}^{0}(v)-\operatorname{dim} K_{1}^{1}(v)=h^{0}(X, E)-h^{1}(X, E)_{\zeta} .
$$

Second step. We have that for $f_{0} \in K_{1}^{0}(v)$, there is $f_{1}$ such that $\bar{\partial}_{E}\left(f_{1}\right)+v f_{0}=0$. We define

$$
v^{(2)}: K_{1}^{0}(v) \rightarrow K_{1}^{1}(v), f_{0} \mapsto\left[v f_{1}\right] .
$$

The $\operatorname{map} v^{(2)}$ is well defined:

(1) $\bar{\partial}_{E}\left(v f_{1}\right)=0 ; v \wedge v f_{1}=0 ;\left[v f_{1}\right] \in H^{1}(X, E)_{\zeta}$.

(2) if $\bar{\partial}_{E}(g)=\bar{\partial}_{E}\left(f_{1}\right)=-v f_{0}$ then $\bar{\partial}_{E}\left(f_{1}-g\right)=0 f_{1}-g=s \in H^{0}(X, E)$; we have $v f_{1}-v g=v s=0$ in $K_{1}^{1}(v)$ since $v s \in \operatorname{Im}\left(v^{(1)}\right)$.

Now if $f_{0} \in \operatorname{ker} v^{(2)}$, there is $f_{2} \in \mathcal{C}^{\infty}(E)$ such that $v f_{1}=-\bar{\partial}_{E} f_{2}-v s$, where $s \in H^{0}(X, E)$, then we have $\bar{\partial}_{E} f_{2}+v\left(f_{1}+s\right)=0$. Let $f_{1}^{\prime}=f_{1}+s$, then

$$
\left(\bar{\partial}_{E}+t v\right) \cdot\left(f_{0}+t f_{1}^{\prime}+t^{2} f_{2}\right)=0 \bmod t^{3} .
$$

We reset the notation by $f_{1}=f_{1}^{\prime}$. Let $K_{2}^{0}(v)=\operatorname{ker} v^{(2)}$ and $K_{2}^{1}(v)=\operatorname{Coker}\left(v^{(2)}\right)$, we have

$$
\operatorname{dim} K_{2}^{0}(v)-\operatorname{dim} K_{2}^{1}(v)=\operatorname{dim} K_{1}^{0}(v)-\operatorname{dim} K_{1}^{1}(v)=h^{0}(E)-h^{1}(E)_{\zeta} .
$$

Inductive Step. For $i \geq 2$, we define inductively the following linear maps:

Let $K_{i}^{0}(v):=\operatorname{ker}\left(v^{(i)}\right), K_{i}^{1}(v):=\operatorname{Coker}\left(v^{(i)}\right)$ and define recursively

$$
v^{(i+1)}: K_{i}^{0}(v) \rightarrow K_{i}^{1}(v), \quad f_{0} \mapsto\left[v f_{i}\right] .
$$

We have that

$$
\operatorname{dim} K_{i+1}^{0}(v)-\operatorname{dim} K_{i+1}^{1}(v)=\operatorname{dim} K_{i}^{0}(v)-\operatorname{dim} K_{i}^{1}(v)=h^{0}(E)-h^{1}(E)_{\zeta} .
$$

As in step 2, the condition to solve (5) at the level $n$ is that $f_{0} \in \operatorname{ker} v^{(n)}$. In fact if $v^{n}\left(f_{0}\right)=0$ we can find $s \in K_{n-1}^{0}(v) \subset H^{0}(X, E)$ such that $v f_{n}=-\bar{\partial}_{E} f_{n+1}-v s$, then letting $f_{n}^{\prime}=f_{n}+s$ we get $\bar{\partial}_{E} f_{n+1}+v f_{n}^{\prime}=0$ when $\bar{\partial}_{E} f_{n}=\bar{\partial}_{E} f_{n}^{\prime}=v f_{n-1}$. Note that we only modify the $n$-term of the sequence $f_{n}$.

Since $K_{i+1}^{0}(v) \subset K_{i}^{0}(v)$ this process stop in a finite number of steps, so we can find an integer $m$ such that the linear map $v^{(m+1)}: K_{m}^{0}(v) \rightarrow K_{m}^{1}(v)$ is the zero map. Then we can find a vector space $K_{m}^{0}(v) \subset H^{0}(X, E)$ such that for any $f_{0} \in K_{m}^{0}(v)$, $\left(\zeta, f_{0}\right)$ allows a linear formal solution. It follows that $K_{m}^{0}(v) \subset W_{\zeta}$. Note that

$$
\operatorname{dim} W_{\zeta} \geq \operatorname{dim} K_{m}^{0}(v) \geq \operatorname{dim} K_{m}^{0}(v)-\operatorname{dim} K_{m}^{1}(v)=h^{0}(E)-h^{1}(E)_{\zeta} .
$$


Now the proof of the Proposition 1.2 is as follows :

Proof. By semicontinuity of $h^{1}(E)_{\zeta}$ as $\zeta$ varies in $H^{1}\left(X, \mathcal{O}_{X}\right)$, it is enough to prove the result for general $\zeta$. For a general element $\zeta \in H^{1}\left(X, \mathcal{O}_{X}\right)$, the analytic curve $\gamma(t)=\exp (t \zeta)$ is Zariski dense in $P$. Letting $k=h^{0}(E)-h^{1}(E)_{\zeta}-1$ we get that for all $t, \gamma(t) \in \mathcal{W}(E)^{k}$. In fact, first we note that $h^{0}(E) \geq k+1$, that is, $\gamma(0)=\mathcal{O}_{X} \in \mathcal{W}(E)^{k}$, so taking an affine open set $U$ of $P$ and polynomial equations $F_{j}$ for the scheme $\mathcal{W}(E)^{k} \cap U$, we get that $F_{j}(\gamma(t))=0 \bmod t^{n}$ because we can deform a space of dimension $\geq k+1 \bmod t^{n}$. Since $F_{j}(\gamma(t))$ is holomorphic and $F_{i}(\gamma(t))=0 \bmod t^{n}$ for all $n$ we get $F_{i}(\gamma(t)) \equiv 0$, that is, $\gamma(t) \in \mathcal{W}(E)^{k}$. Since the image of $\gamma$ is Zariski dense we get that $\mathcal{W}(E)^{k}=P$. This implies $k \leq r_{C}(E)$.

Remark 3.2. In the previous proof, the Artin approximation theorem has not been fully used since $\gamma(t)=\exp (t \zeta)$ is already analytic, using it one can show directly that the sections of the vector bundle deform along $\gamma(t)$. This gives again $\gamma(t) \in \mathcal{W}(E)^{k}$.

\section{Generic VAnishing toward the $\partial \bar{\partial}$ LEMma}

In this section we give a proof of a piece of the generic vanishing of Green and Lazarsfeld (See [7]).

Lemma 4.1. Let $E=\Omega_{X}^{k}$ be and $\omega=f_{0} \in H^{0}(X, E)=H^{k, 0}(X)$ a section. Let $\zeta \in H^{1}\left(X, \mathcal{O}_{X}\right)$ be and assume that $\zeta \cdot \omega=0 \in H^{1}(X, E)=H^{k, 1}(X)$, then $(\zeta, \omega)$ admits a formal linear solution (See definition 2.1).

Proof. Since $H^{1}\left(X, \mathcal{O}_{X}\right) \simeq H^{0,1}(X) \simeq \mathcal{H}^{0,1}(X)$ we can take a representative of $[v]=\zeta$ to be harmonic, so $\partial v=0$ and $\bar{\partial} v=0$. Assume that $[v \wedge \omega]=0$ in $H^{1}\left(X, \Omega_{X}^{k}\right) \simeq H_{\bar{\partial}}^{k, 1}(X)$, then there exists $\alpha \in \operatorname{Im}\left(\bar{\partial}: \mathcal{A}^{k, 0}(X) \rightarrow \mathcal{A}^{k, 1}(X)\right)$ such that $v \wedge f_{0}=\bar{\partial} \alpha$. We have also that $\partial\left(v \wedge f_{0}\right)=\partial(v) \wedge f_{0}+v \wedge \partial \alpha=0+0=0$, then $v \cdot f_{0} \in \operatorname{ker}\left(\partial: \mathcal{A}^{k, 1}(X) \rightarrow \mathcal{A}^{k+1,1}(X)\right)$. Applying the $\partial \bar{\partial}$-lemma (See e.g. [13, Proposition 6.17) we can find $g_{1} \in \mathcal{C}^{\infty}\left(\Omega_{X}^{k-1}\right)$ such that

$$
v \cdot f_{0}=-\bar{\partial} \partial\left(g_{1}\right) \text {. }
$$

Let $f_{1}=\partial g_{1}$ be, we have that $\bar{\partial} f_{1}+v \wedge f_{0}=0$. Suppose that $f_{i}=\partial g_{i}$ such that for $i=1, \ldots, n$, we have $0=\bar{\partial} f_{i}+v \wedge f_{i-1}=\bar{\partial} \partial g_{i}+\partial v \wedge g_{i-1}$. We perform the $(n+1)$ th step. Consider $\theta_{n}=\partial v \wedge g_{n}$. We have $\theta_{n}=\partial\left(v \wedge g_{n}\right)$ and $\bar{\partial}\left(\theta_{n}\right)=-v \bar{\partial} \partial g_{n}=$ $-v \wedge v \wedge \partial g_{n-1}=0$, then by $\partial \bar{\partial}$-lemma we have $\theta_{n} \in \operatorname{ker}(\bar{\partial}) \cap \operatorname{Im}(\partial)=\operatorname{Im}(\partial \bar{\partial})$, thus there is $g_{n+1}$ such that $\theta_{n}=\bar{\partial} \partial\left(g_{n+1}\right)$. Taking $f_{n+1}=\partial g_{n+1}$ we have

$$
(\bar{\partial}+t v)\left(f_{0}+\sum_{i=1}^{\infty} t^{i} \partial g_{i}\right)=0 .
$$

Arguing as before we get

Proposition 4.2. (Green-Lazarsfeld) Let $\omega \in H^{0}\left(X, \Omega_{X}^{k}\right)$ and $\zeta \in H^{1}\left(X, \mathcal{O}_{X}\right)$ be and assume that $\zeta \cdot \omega=0 \in H^{1}\left(X, \Omega_{X}^{k}\right)$. Define $\gamma: \mathbb{C} \rightarrow P, \gamma(t)=\exp t \zeta$, then there is a family of sections $\omega(t) \in H^{0}\left(X, \Omega_{X}^{k}(\gamma(t))\right)$ along $\gamma(t)$ such that $\omega(0)=\omega$.

Remark 4.3. The proof is the same of the one given for the Proposition 1.1. Following Green and Lazarsfed we note that the Zariski closure of $\gamma(t)$ is an abelian subvariety of $P$. If $\zeta$ is linearly very generic, that is, is not contained in a countable union of proper vector subspaces of $H^{1}\left(X, \mathcal{O}_{X}\right)$, then $\overline{\gamma(t)}=P$. Assume $k=n=$ 
$\operatorname{dim} X$, then $\Omega_{X}^{n}=K_{X}$ and $\omega \neq 0$. Let $D(\omega)$ be the divisor of $\omega$. If $\zeta \cdot \omega=0$ and $\zeta$ is linearly very generic we have that $D(\omega) \in \operatorname{Div}^{\kappa}(X)_{\text {main }}$ (See (2)), the main component of the paracanonical system.

\section{The Base Locus of the PARACANONICAL SYSTEM}

In this section we will prove Proposition 1.3 We will use again an antilinear isomorphism $H^{1}\left(X, \mathcal{O}_{X}\right) \simeq \overline{H^{0}\left(X, \Omega_{X}^{1}\right)}$. Thus, given $v \in H^{1}\left(X, \mathcal{O}_{X}\right)$, there exists $\beta \in H^{0}\left(X, \Omega_{X}^{1}\right)$ such that $[\bar{\beta}]=v$. Consider the hermitian pairing $<,>$ $H^{0}\left(X, K_{X}\right) \times H^{0}\left(X, K_{X}\right) \rightarrow \mathbb{C}$ given by

$$
<\omega_{1}, \omega_{2}>=(-i)^{n} \int_{X} \omega_{1} \wedge \bar{\omega}_{2} .
$$

For $\beta \in H^{0}\left(X, \Omega_{X}^{1}\right)$ we have the cup product maps

$$
\begin{gathered}
H^{0}\left(X, \Omega_{X}^{n-1}\right) \stackrel{\wedge \beta}{\longrightarrow} H^{0}\left(X, \Omega_{X}^{n}\right)=H^{0}\left(X, K_{X}\right) . \\
H^{0}\left(X, K_{X}\right) \stackrel{\cdot \bar{\beta}}{\longrightarrow} H^{1}\left(X, K_{X}\right)
\end{gathered}
$$

Lemma 5.1. For every $\beta \in H^{0}\left(X, \Omega_{X}^{1}\right)$ we have $\operatorname{Im}(\wedge \beta)^{\perp}=\operatorname{ker}(\bar{\beta})$.

Proof. We have

$\theta \in \operatorname{Im}(\wedge \beta)^{\perp} \Longleftrightarrow 0=\int_{X} \theta \wedge \overline{\alpha \wedge \beta}=\int_{X}(\theta \wedge \bar{\beta}) \wedge \bar{\alpha}, \forall \alpha \in H^{0}\left(X, \Omega_{X}^{n-1}\right) \stackrel{\text { duality }}{\Longleftrightarrow}$ $\theta \wedge \bar{\beta}=0 \in H^{1}\left(X, \Omega_{X}^{n-1}\right) \Longleftrightarrow \theta \in \operatorname{ker} \bar{\beta}$.

Let $\mathbb{P}\left(H^{0}\left(X, K_{X}\right)\right)=\left|K_{X}\right|$ and $\mathbb{P}=\mathbb{P}\left(H^{1}\left(X, \mathcal{O}_{X}\right)\right)$ the projective spaces of $H^{0}\left(X, K_{X}\right)$ and $H^{1}\left(X, \mathcal{O}_{X}\right)$ respectively. Inside $|K| \times \mathbb{P}$ consider the incidence locus $U \subset$ $\left|K_{X}\right| \times \mathbb{P}$ :

$$
U=\{((s),(\zeta)): \zeta \cdot s=0\}
$$

We have that $U$ is the locus parametrizing pairs $(s, \zeta)$ such that $s \in \operatorname{ker} \zeta$. We have seen in Proposition 4.2 that there are not higher obstructions to deform $s$ in the $\zeta$-direction. Let $\pi_{1}: U \rightarrow\left|K_{X}\right|$ and $\pi_{2}: U \rightarrow \mathbb{P}$ be the projections. We remark that the fibers of $\pi_{2}$ are the projective spaces corresponding to $\operatorname{ker}(\bar{\beta})$. As a consequence of the Generic Vanishing Theorems of Green-Lazarsfeld (See [ 6], 7] and 8 , Lemma 4.2) we have:

Proposition 5.2. Assume that $X$ is a variety of Albanese general type with $\chi\left(K_{X}\right)>$ 0 . We have that $\pi_{2}(U)=\mathbb{P}\left(H^{1}\left(X, \mathcal{O}_{X}\right)\right)$. Let $U_{\text {main }}$ be the unique irreducible component of $U$ that dominates $\mathbb{P}\left(H^{1}\left(X, \mathcal{O}_{X}\right)\right)$, then $\operatorname{dim}\left(U_{\text {main }}\right)=\chi\left(K_{X}\right)+q-2$.

Definition 5.3. We call the variety $Y=\pi_{1}\left(U_{\text {main }}\right) \subset\left|K_{X}\right|$ the locus of deformable canonical divisors. It consists in fact of divisors that deform to the main component

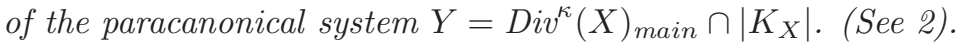

Proposition 5.4. Assume that $X$ is of Albanese general type with $\chi\left(K_{X}\right)>0$. Let $Y$ be the locus of deformable canonical divisors of $X$, then $Y$ is non degenerate, that is, is not contained in any proper linear subspace of $\left|K_{X}\right|$.

Proof. Define $W \subset H^{0}\left(X, K_{X}\right)$ as the subspace

$$
W=\operatorname{span}_{(s) \in Y}\{s\} .
$$

We have to show that $W=H^{0}\left(X, K_{X}\right)$. Suppose by contradiction that $W \subsetneq$ $H^{0}\left(X, K_{X}\right)$, then there exists $\theta$ orthogonal to $\operatorname{ker}(\bar{\beta})$ where $(\bar{\beta})$ is generic, $\bar{\beta} \in$ $H^{1}\left(X, \mathcal{O}_{X}\right)$. 
By lemma (5.1), we have that $\theta \in \operatorname{ker}(\bar{\beta})^{\perp}=\operatorname{Im}(\beta)$. This means that for generic $\beta$,

$$
\theta=\beta \wedge \theta_{\beta}
$$

for some $\theta_{\beta} \in H^{0}\left(X, \Omega_{X}^{n-1}\right)$. Since this is a closed condition, then $\theta \in \operatorname{Im}(\beta)$ for all $\beta$. We can find a point $p \in X, \theta(p) \neq 0$, so we consider the evaluation map

$$
H^{0}\left(X, \Omega_{X}^{1}\right) \otimes \mathcal{O}_{X} \stackrel{e v_{p}}{\longrightarrow}\left(\Omega_{X}^{1}\right)_{p} .
$$

Since $q>n$, there exists $\beta \neq 0$ such that $\beta(p)=0$. Since $\theta=\beta \wedge \theta_{1}$ for some $\theta_{1}$, then $\theta(p)=\beta(p) \wedge \theta_{1}(p)=0$ which is a contradiction, then $W=H^{0}\left(X, K_{X}\right)$.

Now we prove the Proposition 1.3 :

Proof. Consider the base locus $Z_{\kappa}$ of the main component of the paracanonical system:

$$
Z_{\kappa}=\left\{p \in X: p \in D, \forall D \in \operatorname{Div}^{\kappa}(X)_{\text {main }}\right\} .
$$

It follows that $Z_{\kappa} \subset Z=\{p \in X: p \in D(s), \forall(s) \in Y\}$. Since $Y$ is non-degenerate generates $H^{0}\left(X, K_{X}\right)$, then we have $Z=\left\{p \in X: s(p)=0, \forall s \in H^{0}\left(X, K_{X}\right)\right\}=$ $Z_{K}$

\section{REFERENCES}

[1] Arbarello, E; Cornalba, M; P.A. Griffiths, P.A and Harris, J. Geometry of Algebraic curves, Vol. 1. Springer-Verlag, New York, 1985.

[2] Artin, M. On the solution of Analytical Equations. Inventiones Math. 5, 277-291 (1968).

[3] Barja, M.A. Generalized Clifford-Severi Inequalities and the Volume of Irregular Varieties. Duke Math. J. Volume 164, Number 3 (2015), 541-568.

[4] Bloch, S. Semi-Regularity and de Rham Cohomology. Inventiones Math. 17, pp. 51-66(1972).

[5] Clemens, H.; Hacon, C. Deformations of the Trivial Line Bundle and Vanishing Theorems. Inventiones Math. American Journal of Mathematics. Vol 124, No. 4 (Aug., 2002), pp. 769-815.

[6] Green, M.; Lazarsfeld, R. Deformation theory, generic vanishing theorems, and some conjectures o Enriques, Catanese and Beauville. Invent. Math. 90 (1987) 389-407.

[7] Green, M.; Lazarsfeld, R. Higher obstructions to deforming cohomology groups of line bundles. J. Amer. Math. Soc. 4(1991) 87-103.

[8] Mendes-Lopes, M.; Pardini, R.; Pirola, G.P.Continuous families of divisors, paracanonical systems, and a new inequality for varieties of maximal Albanese dimension. Geom Topol. 17, 1205-1223 (2013).

[9] Mendes-Lopes, M.; Pardini, R.; Pirola, G.P. Brill-Noether loci for divisors on irregular varieties. J. Eur. Math. Soc. 16, 2033-2057 (2014).

[10] Mumford, D. Lectures on Curves on an Algebraic Surface. Princeton University Press (1966).

[11] Pareschi, G.; Popa, M. Strong generic vanishing and a higher dimensional Castelnuovo-de Francis inequality. Duke Math. J. Volume 150, Number 2 (2009), 269-285.

[12] Sernesi, E. Deformations of Algebraic Schemes. Grundlehren der Mathematischen Wissenschaften. A Series of Comprehensive Studies in Mathematics. Volume 334. Springer-Verlag Berlin Heidelberg 2006.

[13] Voisin, C. Hodge Theory and Complex Algebraic Geometry I. Cambridge Studies in Advanced Mathematics 76. Cambridge University Press 2002.

[14] Wells, R.O. Jr. Differential analysis on Complex Manifolds. Graduate Texts in Mathematics No. 65, Springer-Verlag, New York- Heidelberg, 2008.

Centro de Ciencias Matemáticas (Universidad Nacional Autonóma de México, Campus Morelia); Apartado Postal 61-3 (Xangari), 58089 Morelia, Michoacán

E-mail address: abel@matmor.unam.mx

Dipartimento di Matematica, Università degli Studi di Pavia, Via Ferrata 1, 27100 Pavia, Italy

E-mail address: gianpietro.pirola@unipv.it 